\title{
Wetting hierarchy in oleophobic 3D electrospun nanofiber networks
}

Urszula Stachewicz ${ }^{1,2,3}$, Russell J. Bailey ${ }^{2}$, Hao Zhang ${ }^{2}$, Corinne A. Stone ${ }^{4}$, Colin R. Willis ${ }^{4}$ and Asa H. Barber ${ }^{1,2,5, *}$

${ }^{1}$ Nanoforce Technology Ltd., Queen Mary University of London, Mile End Road, London E1 4NS, United Kingdom;

${ }^{2}$ Department of Materials, School of Engineering \& Materials Science, Queen Mary University of London, Mile End Road, London E1 4NS, United Kingdom;

${ }^{3} \mathrm{AGH}$ University of Science and Technology, International Centre of Electron Microscopy for Materials Science and Faculty of Metals Engineering and Industrial Computer Science, Al. A. Mickiewicza 30, 30-059 Kraków, Poland

${ }^{4}$ Defence Science \& Technology Laboratory, Porton Down, Salisbury SP4 0JQ, United Kingdom

${ }^{5}$ School of Engineering, University of Portsmouth, Portsmouth PO1 3DJ, United Kingdom

*Correspondence and request for materials should be addressed to A.H.B. (E-mail: asa.barber@port.ac.uk) 


\begin{abstract}
Wetting behavior between electrospun nanofibrous networks and liquids is of critical importance in many applications including filtration and liquid repellent textiles. The relationship between intrinsic nanofiber properties, including surface characteristics, and extrinsic nanofibrous network organization on resultant wetting characteristics of the nanofiber network is shown in this work. Novel 3D imaging exploiting focused ion beam (FIB) microscopy and cryo-scanning electron microscopy (cryo-SEM) highlight a wetting hierarchy that defines liquid interactions with the network. Specifically, small length scale partial wetting between individual electrospun nanofibers and low surface tension liquids, measured both using direct SEM visualization and a nano Wilhelmy balance approach, provides oleophobic surfaces due to the high porosity of electrospun nanofiber networks. These observations conform to a meta-stable Cassie-Baxter regime and are important in defining general rules for understanding the wetting behavior between fibrous solids and low surface tension liquids for omniphobic functionality.
\end{abstract}




\section{Introduction}

The thermodynamically favored spreading of low surface tension liquids on solid surfaces is a significant problem in materials science, especially when contamination of solid surfaces with the low surface tension liquid is to be avoided ${ }^{1}$. Considerable efforts have been made in designing surfaces that are suitable for repelling low surface tension liquids, with a number of promising strategies using nanostructured materials to provide surface oleophobicity for self-cleaning materials ${ }^{2-3}$, enhanced nanofibrous filtration and energy devices ${ }^{4}$, improved biological therapies ${ }^{5}$ and tissue regeneration ${ }^{6-7}$. Electrospun nanofibers have shown considerable success in repelling a range of liquids and chemical modifications are commonly used to tailor their nanofibers surfaces to improve liquid repellency, including coaxial electrospinning to produce Teflon coated electrospun fiber mats ${ }^{8}$ and fluorination of electrospun fibers ${ }^{9}$. Critically, the overall wetting behavior between liquids and nanofiber assemblies is defined by the smaller scale interaction between the nanofibers and contacting liquid. A wetting hierarchy can therefore be considered where the intrinsic wetting behavior between electrospun nanofibers and the contacting liquid defines larger scale wetting behavior that is additionally controlled by extrinsic behavior, specifically the organization of the electrospun fibers contacting the liquid. The demands of repelling low surface tension liquids using electrospun nanofiber networks therefore requires controlling of wetting hierarchy as well as optimized chemical modification of fibrous surfaces. These challenges in relating intrinsic and extrinsic nanofiber properties to overall wetting behavior are non-trivial and have been the subject of intensive research in larger scale textile wetting. Descriptions of fibers repelling liquids were historically developed by Cassie and Baxter ${ }^{10}$ and the application of such descriptions to a range of fibrous networks is currently contentious. Specifically, Cassie-Baxter theory has successfully described wetting of acrylic acid grafted electrospun poly(vinylidene fluoride) (PVDF) fibers with water ${ }^{11}$ but is insufficient for predicting wetting of perfluoroalkyl ethyl methacrylate (PPFEMA) coated poly(caprolactone) (PCL) with low surface tension liquids including $n$-decane, $n$-octane, and $n$-heptane ${ }^{12}$. The inconsistencies in applying Cassie-Baxter theory to oleophobic electrospun nanofiber networks, despite observations of Cassie-Baxter regimes where the liquid is nonspreading at the network surface ${ }^{13}$, thus require quantitative descriptions of the wetting behavior. Perhaps the only attempts to fully describe wetting of fibers was presented for a 
simple system of two parallel glass fibers contacting a low surface tension oil droplet ${ }^{14}$. A consideration of the anisotropy of the fibers was shown to provide a liquid repellent regime for the system, despite oil completely wetting glass surfaces. Thus, wetting behavior between fibers and liquids requires understanding of the intrinsic fiber properties as well as extrinsic properties of the network. This wetting hierarchy will be critical in oil repellent electrospun nanofiber networks but requires evaluation of wetting at nanofiber length scales as well as considering the complex $3 \mathrm{D}$ organization of these nanofibers within the network.

A conclusive description of low surface tension liquid repellency at electrospun nanofiber surfaces is presented here using novel microscopy techniques to understand wetting from the nanoscale to the larger 3D assembles of the fibrous network. These approaches allow us to comprehensively describe liquid interactions with the electrospun nanofiber network and identify the importance of a wetting hierarchy at multiple length scales as shown in Figure 1. Such approaches are broad and important in future liquid repellent strategies using the electrospun nanofibers of this work as well as generally controlling wetting behavior using nanofiber networks.

The first critical evaluation of liquid droplet interactions with fibers was conducted by Cassie and Baxter. ${ }^{10}$ This paper is frequently employed in an unmodified fashion due to the effectiveness of the 'Cassie-Baxter equation' in describing a liquid droplet wetting contact angle $\theta_{C B}$ on a fibrous network, thus containing pores, using the relationship ${ }^{15-17}$ :

$$
\cos \theta_{C B}=f_{1} \cos \theta_{Y}-f_{2}
$$

where $\theta_{Y}$ is the Young contact angle of liquid on a fiber, $f_{1}$ is the total solid-liquid interfacial area and $f_{2}$ is the liquid-air interfacial area at the contact plane with the liquid droplets $\left(f_{1}+\right.$ $f_{2}=1$ ). While Cassie-Baxter theory has been exploited to explain a range of liquid contact angles with fibrous materials, the original work critically examined a liquid contacting fibers as a 2-dimensional problem. Indeed, the Cassie-Baxter model can be more accurately described as a liquid droplet contacting solid spheres when considered in a three- 
dimensional context. The anisotropy of fibrous structures, especially when liquid droplet sizes approach the contact fiber diameters, is thus ignored. Recent work has additionally highlighted the need to understand the ingress of liquids within electrospun fibrous mats, ${ }^{18-}$ ${ }^{20}$ with perhaps the aim of justifying the use of Cassie-Baxter models through observation of liquid-air interfaces. In particular, optical imaging of liquids with fluorescent dyes at electrospun fiber mat surfaces suggested little penetration of the liquid. ${ }^{20}$ Thus, the current literature is somewhat diverse in both evaluating the wetting behavior between liquids and electrospun fiber surfaces, as well as indicating if electrospun fibers are effective at liquid repellency. The more rigorous evaluations ${ }^{8}$ suggest improvement in the superhydrophobicity and oleophobicity of fluorinated electrospun nanofiber mats but recent investigations into assessing liquid penetration into the electrospun fibrous mat ${ }^{20}$ require further evaluation. The literature would therefore prompt open questions about the nature of the liquid-solid interface when considering electrospun nanofibers, where the fiber diameter is often in the range of a few hundreds of nanometers and beyond the optical resolution of conventional light microscopy, and about the ability of the CassieBaxter equation to describe liquid contact angles with electrospun, or indeed any anisotropic nanofibrous system. Experiments are performed in this study to evaluate the apparent contact angle between a low surface tension liquid and electrospun nanofiber networks at a range of length scales. Contact angles measurements throughout this work therefore refer to the observed apparent contact angle. Extrinsic effects require the evaluation of the interaction between the electrospun nanofiber 3D network and low surface tension liquid whereas intrinsic behavior warrants experimental design examining individual nanofiber wetting with the liquid that ignores organization effects.

\section{Results}

A nanofibrous network was electrospun from a solution of polyamide and plasma treated with fluoropolymer for enhanced oil repellency. The quality of plasma coating was verified with X-ray photoelectron spectroscopy (XPS) (see Supporting Information, Figure S1). The wetting behavior at the individual electrospun nanofiber length scale was evaluated using a nanofiber Wilhelmy balance experiment and larger 3D network organization wetting characterized using cryo- FIB-SEM imaging. This latter technique provides 3D imaging 
capability to determine the spreading of liquid throughout the nanofibrous network and is critical in supporting theoretical descriptions that assume particular liquid spreading regimes.

\section{Individual nanofiber wetting behavior}

A Wilhelmy balance method was used to quantify the contact angle between a low surface tension oil droplet with an individual electrospun nanofiber attached to an atomic force microscope (AFM) probe as shown in Figure 2. The wetting force acting between individual electrospun nanofibers and liquid oil in air with experimental time is shown in Figure 2. The force curve shows a distinct increase in the wetting force, which corresponds to the nanofiber moving into contact with the liquid and partial spreading of the oil over the nanofiber surface. This resultant spreading exerts a force on the nanofiber surface that is measurable with the AFM. The magnitude of the wetting force acting on the individual nanofiber is determined by the contact angle made with the nanofiber surface. This contact angle $\theta_{\text {nano }}$ is given by the force balance:

$$
F=\pi \gamma_{P D M S} \cos \theta_{\text {nano }} d
$$

where $d$ is the nanofiber diameter measured from SEM images, $\gamma_{P D M S}$ is surface energy of

silicone oil equal to $20 \mathrm{~mJ} \cdot \mathrm{m}^{-2}$ and $\theta_{\text {nano }}$ is the liquid-nanofiber wetting contact angle. We note that $\theta_{\text {nano }}$ is effectively $\theta_{Y}$ as both consider liquids contacting a continuum surface, although the former requires small scale measurement. Resultant contact angles between the individual electrospun nanofibers and liquid oil are shown in Table 1 and indicates partial wetting contact angles of $60^{\circ}$.

\section{D imaging of nanofiber networks}

Electrospun nanofiber networks supporting liquid oil droplets were imaged using cryo-FIBSEM with an example of serial 2D images reconstructed into a complete 3D image shown in Figure 3. Complementary 2D cryo-SEM images were additionally used to examine the wetting behavior between the oil and electrospun fibers, especially as the wetting between 
the liquid droplet edge and nanofiber surfaces was observed to be potentially different to the larger scale wetting of the whole oil droplet in contact with the fibrous array as shown in Figures 4 and 5 . The volume fraction of nanofibers within the 3D network was measured from FIB-SEM images as shown in Figure 3 and was given as $0.04^{21}$. Resultant oil contact angles with the nanofibers were taken from 3D imaging and verified using conventional contact angle measurements employing optical microscopy (see Supporting Information, Figure S2 and Table S1) to give a macroscopic oil-network contact angle $\theta_{\text {macro }}$ of $110 \pm 7^{\circ}$. Critically, the 3D imaging highlighted a liquid spreading that was confined to the surface of the electrospun nanofiber network, with no observable ingress of oil into the network. As the electron microscopy imaging is sufficient to resolve the liquid with nanometer resolution, we are confident that the network is repelling the oil. Wetting behavior between the solid and oil is thus defined by the electrospun nanofibers at the surface of the 3D network.

\section{Discussion}

Macroscopic contact angle measurements between oil droplets and plasma treated electrospun nanofiber networks indicate non wetting behavior, as shown with both 3D SEM imaging and optical microscopy. The oil contact angle on the fibrous network is higher than the contact angle on a plasma treated glass slide (which is smooth and planar - see Supporting Information, Figures S2 and S3 and Tables S1 and S2). Oil droplets wet uncoated electrospun PA6 nanofibers rapidly, as presented in Figure S6 of the Supporting Information. Uncoated nanofibers appear to be completely wetted by the oil and is assumed to have a resultant $0^{\circ}$ contact angle. Indeed, comparable measurements on PA6 films on glass slides without plasma treatment exhibit low contact angles tending towards complete wetting (see Supporting Information, Figure S7 and Table S3). A series of wetting experiments were carried out to quantify $\theta_{\text {macro }}$ between silicone oil and plasma treated electrospun fiber arrays using conventional observations of contact angle using optical microscopy. A typical optical image of the oil contact angle with the electrospun fiber array is shown in Supporting Information, Figure S2, and highlights a non-wetting condition providing a contact angle of $110 \pm 7^{\circ}$. The organization of electrospun nanofibers therefore provides oleophobicity that is enhanced compared to a macroscopic angle of $72.6^{\circ}$ oil on a plasma coated glass slide. 
We can conclude that the extrinsic nanofibrous network geometry is able to provide oleophobicity beyond the influence of fluorinated surface chemistry. 3D imaging provides higher spatial resolution information beyond optical microscopy, which indicates intrinsic effects related to the wetting between individual nanofibers and the contacting oil as shown in Figures 5(b), (c) and (d). The size of oil droplets has been previously shown to dictate wetting behavior between two parallel fibers ${ }^{14}$. Specifically, smaller droplet volumes provide wetting regimes whereas increasing droplet volume above a critical volume promotes a non-wetting condition. Evidence of sprayed oil droplets wetting electrospun nanofibers was absent in all high resolution electron microscopy evaluations as shown in Figures 3-6. We therefore conclude that the oil droplet sizes used in this study are above the critical volumes needed to wet the electrospun nanofibers.

Quantitative descriptions of intrinsic wetting behavior were made using both Wilhelmy balance methods and cryo-SEM imaging in Figure 6 and highlighted partial wetting between the oil and electrospun nanofibers, with a contact angle of $\theta_{\text {nano }}=60^{\circ}$. This nansocale contact angle differs from the larger scale contact angle measurements of $110^{\circ}$ and critically indicates the wetting hierarchy from large scale to small scale. The electrospun nanofiber contact angle was additionally lower than for the plasma coated slide, suggesting a wetting variability due to the cylindrical curved surface of an individual nanofiber compared to the planar slide ${ }^{22}$. Thermodynamic arguments have been used to show that fiber curvature influences liquid spreading power relative to a flat planar surface ${ }^{23}$. In addition, surface free energy of electrospun nanofibers have been shown to differ from bulk behavior but the typical range of electrospun nanofiber diameters is too limited to provide any surface free energy variation ${ }^{24-25}$. The observed hierarchical wetting where a contact angle between liquid and individual nanofibers differs from a larger scale multi-fiber contact with a macroscopic liquid droplet has been additionally used in previous literature, including liquid water contacting silicon micropillars imaged using environmental scanning electron microscopy ${ }^{26}$. 3D wetting using cryo-FIB-SEM at nanoscale was also investigated for water and silicon nanowires where complex wetting with locally bent droplets at substrate interfaces was observed ${ }^{27}$.

To describe hierarchical wetting conclusively, Cassie-Baxter theory is applied to our intrinsic contact angle data to give a predicted macroscopic contact angle as shown in Figure 7 using 
the directly measured porosity of nanofiber mats, $f_{1}=0.04$, from 3D imaging ${ }^{21}$. Further models have been developed to modify Cassie-Baxter theory and should also be considered when describing wetting between the oil droplets and the electrospun nanofibrous network. Specifically, a meta-stable Cassie-Baxter model has been additionally proposed to predict super-oleophobicity of non-woven fabrics of relatively large diameter textile fibers ${ }^{28}$. A meta-stable Cassie-Baxter describes liquid sitting on the surface of a fibrous network with air pockets created by the surface roughness, but is effective at describing entangled random roughness surfaces as opposed to the regular porosities considered by a standard Cassie-Baxter regime. Indeed, the application of Cassie-Baxter to compositional or nonuniform surfaces has been shown to be problematic ${ }^{29,30}$. Thus, a macroscopic contact angle between an oil droplet and the irregular electrospun nanofiber network, $\theta_{m C B}$, can be defined from a meta-stable Cassie-Baxter consideration using ${ }^{28}$ :

$\cos \theta_{m C B}=\frac{R\left(\pi-\theta_{Y}\right)}{d+R} \cos \theta_{\text {nano }}+\frac{R}{d+R} \sin \theta_{\text {nano }}-1$

where $R$ is the nanofiber radius and $2 d$ is the distance between two adjacent fibers in the network. The average nanofiber radius is calculated based on fiber diameter distribution plots taken from SEM imaging, see Supporting Information, page S7 Figure S4 and give a value of $R=0.4 \mu \mathrm{m}$ for the electrospun network. The $2 d$ value is taken from the previously calculated average distance of $1.7 \mu \mathrm{m}$ between nanofibers from the $3 \mathrm{D}$ reconstruction of electrospun PA6 nanofiber networks ${ }^{21}$. The 3D images of oils droplets on the nanofiber network, as shown in Figure 3, importantly indicate a lack of oil penetration within the network. Such an observation is counter to previous literature where 3D imaging showed partial penetration of liquid water within networks of silicon nanowire-type pillars ${ }^{27}$. Thus, a Cassie-Baxter condition where the oil droplet is situated at the network surface is found.

The standard Cassie-Baxter and meta-stable Cassie-Baxter models were used to predict the extrinsic macroscopic contact angle of the oil droplet on the nanofiber network using the organization of the nanofibers, which defines the volume fraction of nanofibers in contact with the oil droplet, and the intrinsic contacting angle between the oil and individual nanofibers. Figure 7 shows the predicted macroscopic contact angles for a range of intrinsic 
contact angles for the Cassie-Baxter and meta-stable Cassie-Baxter theories. Both theories predict an increasing macroscopic oil contact angle as the intrinsic contact angle between the oil and nanofiber surfaces increases. However, the meta-stable Cassie-Baxter model exhibits a larger increase in macroscopic contact angle as the intrinsic contact angle between the oil and nanofiber increase compared to the Cassie-Baxter model. Our experimental observations can be used to confirm the applicability of each model to our oil droplet contacting plasma treated electrospun nanofibers. The intrinsic contact angle of $60^{\circ}$ allows the prediction of macroscopic contact angle as shown in Figure 7. Critically, the metastable Cassie-Baxter model provides a predicted macroscopic contact angle of $113^{\circ}$, which correlated precisely with our experimental observations (see Figure S2 in Supporting Information), whereas Cassie-Baxter considerably overestimates the macroscopic contact angle at approximately $160^{\circ}$. The Cassie-Baxter model considers a rigid network and does not fit the data whereas the meta-stable Cassie-Baxter predicts wetting behavior. The issue of a liquid droplet causing a deformation of fibers must therefore be a suitable mechanism that defines wetting. Electrospun fibers are typically of high aspect ratio so relatively easy to deflect under the action of the oil droplet. Network sagging under a liquid droplet is therefore suggested as the mechanism responsible for the macroscopic wetting behavior between the liquid and electrospun nanofiber network ${ }^{31}$. These results highlight both the importance of experimentally linking the small scale intrinsic wetting behavior to larger scale extrinsic contact angles for electrospun nanofibrous networks and the suitability of the meta-stable Cassie-Baxter in accurately predicting this resultant wetting for low surface tension liquids.

Finally, our results predict superoleophobicity $\left(\theta_{\text {macro }}>150^{\circ}\right)$ for electrospun nanofibrous networks only when the intrinsic contact angle between the oil and nanofiber exceeds $\theta_{\text {nano }}$ values of $120^{\circ}$. However, $120^{\circ}$ is unlikely to be achievable other than for relatively high surface tension organic liquids. Plasma coating with highly fluorinated $\mathrm{C}_{8} \mathrm{~F}_{17}$ chains as used in this work is a limiting factor as further oil repellent chemistry is difficult to achieve, in addition to a drive by industry to use shorter chains with potentially poorer olephobicity ${ }^{32-}$ 33. Network design and organization of electrospun nanofibers therefore becomes more dominant in achieving superolephobic surfaces. 


\section{Conclusions}

The hierarchical wetting behavior between a low surface tension oil and fluorinated electrospun nanofiber networks from nanoscale to macroscopic length scales was conclusively evaluated using a range of microscopy techniques. Oil partially wetting individual electrospun nanofibers in conjunction with extrinsic nanofiber organization was highlighted as defining larger scale wetting behavior and is described fully using a metastable Cassie-Baxter condition. Such intrinsic partial wetting $\left(\theta_{\text {nano }}=60^{\circ}\right)$ between oils and fluorinated electrospun nanofibers are still able to give extrinsic non-wetting conditions $\left(\theta_{\text {macro }}=110^{\circ}\right)$ with relatively large oil droplets due to the inherent high porosity of electrospun nanofibrous networks. These results show the synergy between surface chemistry and extrinsic organization in electrospun nanofiber networks for enhanced olephobic properties in a material system.

Methods (less than 3000 words)

\section{Electrospinning of PA6 nanofibers}

Polyamide 6 (PA6, $M_{\mathrm{w}}=24,000 \mathrm{~g} \cdot \mathrm{mol}^{-1}$, BASF, Ultramid B33 L, Germany) was dissolved in a mixture of acetic acid ( $\geq 99.7 \%$, Sigma Aldrich, U.S.A.) and formic acid (98\%, Sigma Aldrich, U.S.A.) (50/50 mass ratio) to produce a resultant polymer concentration of $12 \mathrm{wt} \%$ in solution. The PA6 polymer solution was electrospun into nanofibers using a large scale multi-jet electrospinning setup (NanoSpider, Elmarco, Czech Republic). The experimental settings are as published previously ${ }^{21,34}$.

\section{Attachment of individual electrospun nanofibers to AFM tips}

The attachment of individual electrospun nanofibers to the apex of an AFM tip was carried out in the SEM containing a custom built nanomanipulator (attoAFM II, attocube GmbH, Germany) according to previous methodologies ${ }^{35-36}$. A small section of the electrospun PA6 mat was placed on carbon tape and attached to a sample stage holder within the SEM chamber. A small droplet of vacuum compatible glue (Poxipol, Argentina) was also added to 
the sample stage holder. An AFM tip (Veeco, U.S.A., spring constant $K=0.2 \mathrm{~N} . \mathrm{m}^{-1}$ ) was attached to the nanomanipulator system and the tip translated towards the glue while observing using the SEM until contact with the glue occurred. Residual glue remained at the apex of the AFM tip after retraction. Subsequent translation and contact between the AFM tip and an individual PA6 nanofiber within an electrospun mat was carried out. Following solidification of the glue, a FIB system integrated within the SEM was used to cut the nanofiber and leave a nanofiber length of approximately $10 \mu \mathrm{m}$ fixed to the AFM tip as shown in Figure 2. The diameter of electrospun PA6 nanofibers attached to the AFM probe was typically $400-500 \mathrm{~nm}$.

\section{Plasma treatment}

Electrospun PA6 fiber mats and glass slides were used in their as-manufactured state but also surface modified to provide a more hydrophobic, and potentially oleophobic, material. Surface modification of the PA6 fiber sample was achieved using plasma treatment to add fluoropolymer to the electrospun fiber surface as described previously ${ }^{37}$ to repel low surface tension liquids. The treatments of electrospun fibers were carried out in a inductively coupled glass cylindrical glow discharge reactor, $0.01 \mathrm{~m}$ diameter, $4.3 \times 10^{-3} \mathrm{~m}^{3}$ volume, base pressure of $1 \times 10^{-2}$ mbar. The reactor was connected to a two stage Edwards rotary pump via a liquid nitrogen cold trap with a thermocouple pressure gauge inline. A monomer tube containing $1 \mathrm{H}, 1 \mathrm{H}, 2 \mathrm{H}, 2 \mathrm{H}$-perfluorodecyl acrylate (PFAC8, Fluorochem, U.K.) was purified by freeze-thaw cycles prior to use and attached to the air inlet side of the reactor. The deposition apparatus was heated to approximately $32{ }^{\circ} \mathrm{C}$ in order to generate a stable vapor pressure. Once base pressure had been reached, the monomer vapor was introduced into the reactor. The reactor was purged with the vapor for five minutes, and once the pressure had stabilised at between $8 \times 10^{-2}$ and $1 \times 10^{-2}$ mbar, a radio frequency (RF) generator was switched on to create a $40 \mathrm{~W}$ continuous wave plasma. This was allowed to run for 30 seconds. At this point the pulse generator was turned on, at a pulsing sequence of $40 \mu$ s on, $20 \mathrm{~ms}$ off. Once the plasma deposition had recovered, as indicated by an input power of 40 $\mathrm{W}$ and a stable pulse envelope, confirmed using an oscilloscope the deposition was allowed to run for 20 minutes. At the end of the treatment the RF generator was switched off and the reactor purged for 2 minutes with monomer vapor, prior to being evacuated back to 
base pressure. Once base pressure was reached the vacuum chamber was isolated from the pump and the system was brought up to atmospheric pressure and the samples were removed. All wetting experiments are restricted to the AFM tips where the plasma treatment was applied after the attachment in order to avoid any removal of plasma coating when FIB was used. The plasma is stable over a calculated length of $74 \mu \mathrm{m}^{38}$, known as the Debye length, which indicates the distance at which the coverage of the fluorinated coating will be uniform within the electrospun fibrous mat.

Contact angle measurements on individual nanofibers.

The wetting behavior of the individual electrospun PA6 nanofibers was carried out according to Wilhelmy balance experiments ${ }^{24-25,39}$ in air using a stand-alone AFM (NT-MDT NTegra, Russia) with the electrospun PA6 nanofiber-AFM tips replacing conventional AFM tips. The experiments were performed at $22{ }^{\circ} \mathrm{C}$ and a humidity of $35 \%$. A probe liquid of silicone oil was placed separately into a liquid cell situated below the fiber-AFM tip. Contact between the liquid surface and the individual PA6 nanofiber attached to the AFM tip was achieved by first moving the liquid cell up towards the nanofiber in a standard AFM landing mode. Proximity of the liquid surface to the PA6 nanofiber was determined using the AFM in semi-contact mode by an cantilever amplitude drop. The AFM was then switched to contact mode and the liquid cell moved up more slowly using the z-piezo positioner of the AFM, situated underneath the liquid cell, until contact of the PA6 nanofiber with the liquid surface was achieved, determined as an abrupt bending of the AFM cantilever down towards the liquid as wetting of the nanofiber surface occurred. The approach velocity was of the order of $1 \mu \mathrm{m} \cdot \mathrm{s}^{-1}$

The change in the AFM cantilever deflection during the progression of the experiment is shown in Figure 2. The cantilever deflection was recorded during the partial immersion of the nanofiber length within each probe liquid and the nanofiber was removed by lowering the liquid cell using the AFM z-piezo positioner. Cantilever deflection was converted to force by first determining the cantilever spring constant $K$, calibrated using the thermal noise method ${ }^{40}$. The displacement of the AFM cantilever, measured as the deflection signal $x$, was converted to force $F$ acting on the nanofiber using $F=K x$. 
$X$-ray photoelectron spectroscopy

The chemistry of the electrospun plasma treated PA6 nanofiber surfaces was analyzed with the parallel acquisition X-ray photoelectron spectroscopy (PARXPS) (Thermo Scientific Theta Probe, U.S.A.) collecting angle resolved data over a $60^{\circ}$ range of angles in parallel, without the need to tilt the sample, (see Supporting Information, Figures S1). The chemistry of nanofibers is verified up to $10 \mathrm{~nm}$ in high vacuum conditions. Using parallel acquisition of angular data in PARXPS, the compensation conditions are the same for all angles and any changes in the spectra as a function of angle reflect real chemical differences. XPS was performed over an area of $700 \times 300 \mu \mathrm{m}$ and elemental compositions of carbon, oxygen, nitrogen and fluorine corresponding to polar side groups in the polyamide chain and the plasma coating were recorded.

\section{Cryo-SEM sample preparation and imaging}

Droplets of silicone oil (Baysilone oil M1000, polydimethylsiloxane, PDMS, Sigma Aldrich, U.K.) were deposited using a spray brush and air compressor (Iwata, Japan) at a pressure of $0.28 \mathrm{MPa}$ and distance $0.3 \mathrm{~m}$ onto electrospun nanofiber mats and flash frozen under liquid nitrogen. The distribution of droplet sizes were known, based on measuring droplet volumes sprayed onto continuum solid surfaces, and ranged from a few microns to $80 \mu \mathrm{m}$ in diameter with a median diameter of $10 \mu \mathrm{m}$ (see droplet size distribution histogram in Supporting Information, Figure S5). The frozen liquid droplets on the nanofiber mat were vacuum transferred and sputter coated with $\mathrm{Au} / \mathrm{Pd}$ for 2-4 min. As samples contained liquid phase agents, all samples were installed into a cryo-stage (Gatan, U.K.) incorporated within the FIB-SEM chamber. The cryo-stage allows liquid nitrogen to be pumped through a sample holder containing the electrospun fiber mat with droplets, and maintains low temperatures from -130 to $-160{ }^{\circ} \mathrm{C}$ in this work. The liquid droplets on the electrospun nanofiber mat are therefore in the solid state when introduced into the vacuum chamber, thus preserving the shape of the liquid droplet and allowing high resolution SEM imaging of the liquid interaction with the solid fibrous mat while suppressing liquid flow or evaporation. SEM 
imaging was taken at a working distance of $0.1 \mathrm{~m}, 5 \mathrm{kV}$ electron beam accelerating voltage and beam current no higher than $53.3 \mathrm{pA}$. The sample stage was tilted at $52^{\circ}$ to measure contact angle and observe the contact line between silicone oil and nanofibers. The measurement of contact angle on the individual nanofibers from SEM images was performed according to previously published protocols ${ }^{22}$.

\section{Cryo-FIB-SEM work and slice and view procedure}

Visualization of wetting between liquid droplets and electrospun nanofiber mats was achieved using a Small Dual Beam (SDB) microscope (Quanta 3D FEG, FEI Company, E.U./U.S.A.) and following previous protocols ${ }^{21,41}$. The sample stage was tilted within the SDB so that the sample surface was perpendicular to the FIB direction and the electron beam had an angle of $52^{\circ} 36,42$. The SDB allows both imaging of surfaces with SEM and removal of the surface layer using FIB to allow further SEM imaging of sub-surface structure. Gallium ions from FIB impinge onto the sample and cause sputtering of sample fragments, thus allowing section of materials. FIB was performed using an ion beam current from 0.5 $\mathrm{nA}$ to $30 \mathrm{nA}$ accelerated at a voltage of 16 and $30 \mathrm{kV}$ to remove cross-sectional slices of 100 $\mathrm{nm}$. The collected SEM images during FIB sectioning were filtered and colored using Image J (version 1.46r, NIH, U.S.A.) with the electrospun nanofibers and silicone oil droplets reconstructed. The 3D reconstruction of the nanofiber sample with droplets was obtained using Resolve RT (version 5.2 - FEI Edition, Germany) as shown in Figure 3.

\section{Measurement of macroscopic contact angle}

Macroscopic contact angle measurements of silicone oil and plasma treated PA6 electrospun nanofibers and glass slides were taken using a Drop Shape Analysis System (Krüs, DSA100, Germany) (see example in Supporting Information in Figure S2, S3 and S7). The experiments were performed at a temperature of $24{ }^{\circ} \mathrm{C}$. The errors on the measured contact angles were determined based on the standard deviation between contact angles measurements taken from all directions.

\section{Acknowledgments}


This research was supported by the Centre for Defence Enterprise, project number CDE20575. We thank Z. Luklinska at the Queen Mary NanoVision Centre for assistance with microscopy facilities. X-ray photoelectron data were obtained at the National EPSRC XPS User's Service (NEXUS) at Newcastle University, an EPSRC Mid-Range Facility.

\section{Author Contributions}

U.S. and A.H.B proposed the research project.

U.S., R.J.B., H.Z., A.H.B, C.A.S. and C.R.W. carried out the experiments.

U.S. and A.H.B wrote the manuscript.

All authors discussed the results and contributed to the manuscript.

\section{References}

(1) Wong, T.-S.; Kang, S. H.; Tang, S. K. Y.; Smythe, E. J.; Hatton, B. D.; Grinthal, A.; Aizenberg, J. Bioinspired Self-Repairing Slippery Surfaces with Pressure-Stable Omniphobicity. Nature 2011, 477, 443-447.

(2) Liu, K.; Jiang, L. Bio-Inspired Design of Multiscale Structures for Function Integration. Nano Today 2011, 6, 155-175.

(3) Sas, I.; Gorga, R. E.; Joines, J. A.; Thoney, K. A. Literature Review on Superhydrophobic Self-Cleaning Surfaces Produced by Electrospinning. J. Polym. Sci., Part B: Polym. Phys. 2012, 50, 824-845.

(4) Thavasi, V.; Singh, G.; Ramakrishna, S. Electrospun Nanofibers in Energy and Environmental Applications. Energ. Environ. Sci. 2008, 1, 205-221.

(5) Hakimi, O.; Murphy, R.; Stachewicz, U.; Hislop, S.; Carr, A. J. An Electrospun Polydioxanone Patch for the Localisation of Biological Therapies During Tendon Repair. Eur. Cells Mater. 2012, 24, 344-57.

(6) Gomez-Sanchez, C.; Kowalczyk, T.; Ruiz De Eguino, G.; Lopez-Arraiza, A.; Infante, A.; Rodriguez, C. I.; Kowalewski, T. A.; Sarrionandia, M.; Aurrekoetxea, J. Electrospinning of Poly(Lactic Acid)/Polyhedral Oligomeric Silsesquioxane Nanocomposites and Their Potential in Chondrogenic Tissue Regeneration. J. Biomater. Sci. Polym. Ed. 2014, 25, 802-25. 
(7) Kloskowski, T.; Kowalczyk, T.; Nowacki, M.; Drewa, T. Tissue Engineering and Ureter Regeneration: Is It Possible? Int. J. Artif. Organs 2013, 36, 392-405.

(8) Han, D.; Steckl, A. J. Superhydrophobic and Oleophobic Fibers by Coaxial Electrospinning. Langmuir 2009, 25, 9454-9462.

(9) Thorvaldsson, A.; Edvinsson, P.; Glantz, A.; Rodriguez, K.; Walkenstrom, P.; Gatenholm, P. Superhydrophobic Behaviour of Plasma Modified Electrospun Cellulose NanofiberCoated Microfibers. Cellulose 2012, 19, 1743-1748.

(10) Cassie, A. B. D.; Baxter, S. Wettability of Porous Surfaces. Trans. Faraday Soc. 1944, 40, 546-551.

(11) Huang, F. L.; Wang, Q. Q.; Wei, Q. F.; Gao, W. D.; Shou, H. Y.; Jiang, S. D. Dynamic Wettability and Contact Angles of Poly(Vinylidene Fluoride) Nanofiber Membranes Grafted with Acrylic Acid. Express Polym. Lett. 2010, 4, 551-558.

(12) Ma, M. L.; Mao, Y.; Gupta, M.; Gleason, K. K.; Rutledge, G. C. Superhydrophobic Fabrics Produced by Electrospinning and Chemical Vapor Deposition. Macromolecules 2005, 38, 9742-9748.

(13) Tuteja, A.; Choi, W.; Ma, M. L.; Mabry, J. M.; Mazzella, S. A.; Rutledge, G. C.; McKinley, G. H.; Cohen, R. E. Designing Superoleophobic Surfaces. Science 2007, 318, 1618-1622.

(14) Duprat, C.; Protiere, S.; Beebe, A. Y.; Stone, H. A. Wetting of Flexible Fibre Arrays. Nature 2012, 482, 510-513.

(15) McHale, G. Cassie and Wenzel: Were They Really So Wrong? Langmuir 2007, 23, 82008205.

(16) Gao, L. C.; McCarthy, T. J. How Wenzel and Cassie Were Wrong. Langmuir 2007, 23, 3762-3765.

(17) Nosonovsky, M. On the Range of Applicability of the Wenzel and Cassie Equations. Langmuir 2007, 23, 9919-9920.

(18) Liu, Y.; Wu, N.; Wei, Q.; Cai, Y.; Wei, A. Wetting Behavior of Electrospun Poly(L-Lactic Acid)/Poly(Vinyl Alcohol) Composite Nonwovens. J. Appl. Polym. Sci. 2008, 110, 31723177.

(19) Pisuchpen, T.; Chaim-ngoen, N.; Intasanta, N.; Supaphol, P.; Hoven, V. P. Tuning Hydrophobicity and Water Adhesion by Electrospinning and Silanization. Langmuir 2011, 27, 3654-3661. 
(20) Jabal, J. M. F.; McGarry, L.; Sobczyk, A.; Aston, D. E. Wettability of Electrospun Poly(Vinylpyrrolidone) -Titania Fiber Mats on Glass and Ito Substrates in Aqueous Media. ACS Appl. Mater. Interfaces 2009, 1, 2325-2331.

(21) Stachewicz, U.; Modaresifar, F.; Bailey, R. J.; Peijs, T.; Barber, A. H. Manufacture of Void-Free Electrospun Polymer Nanofiber Composites with Optimized Mechanical Properties. ACS Appl. Mater. Interfaces 2012, 4, 2577-2582.

(22) McHale, G.; Rowan, S. M.; Newton, M. I.; Kab, N. A. Estimation of Contact Angles on Fibers. J. Adhes. Sci. Technol. 1999, 13, 1457-1469.

(23) McHale, G.; Käb, N. A.; Newton, M. I.; Rowan, S. M. Wetting of a High-Energy Fiber Surface. J. Colloid Interf. Sci. 1997, 186, 453-461.

(24) Stachewicz, U.; Stone, C. A.; Willis, C. R.; Barber, A. H. Charge Assisted Tailoring of Chemical Functionality at Electrospun Nanofiber Surfaces. J. Mater. Chem. 2012, 22, 22935-22941.

(25) Stachewicz, U.; Barber, A. H. Enhanced Wetting Behavior at Electrospun Polyamide Nanofiber Surfaces. Langmuir 2011, 27, 3024-3029.

(26) Paxson, A. T.; Varanasi, K. K. Self-Similarity of Contact Line Depinning from Textured Surfaces. Nat. Commun. 2013, 4, 1492.

(27) Rykaczewski, K.; Landin, T.; Walker, M. L.; Scott, J. H. J.; Varanasi, K. K. Direct Imaging of Complex Nano- to Microscale Interfaces Involving Solid, Liquid, and Gas Phases. ACS Nano 2012, 6, 9326-9334.

(28) Lee, H.; Willis, C.; Stone, C. Modeling and Preparation of a Super-Oleophobic NonWoven Fabric. J. Mater. Sci. 2011, 46, 3907-3913.

(29) Erbil, H. Y.; Cansoy, C. E. Range of Applicability of the Wenzel and Cassie-Baxter Equations for Superhydrophobic Surfaces. Langmuir 2009, 25, 14135-14145.

(30) Stachewicz, U.; Benett, C.; Barber, A. H. Wetting of Polyamide Film Surfaces with Electrospun Nanofibers. In Multifunctional Polymer-Based Materials; Lendlein, A.,Behl, M.,Feng, Y.,Guan, Z., Xie, T., Eds.; 2012; 47-51.

(31) Konosu, Y.; Matsumoto, H.; Tsuboi, K.; Minagawa, M.; Tanioka, A. Enhancing the Effect of the Nanofiber Network Structure on Thermoresponsive Wettability Switching. Langmuir 2011, 27, 14716-14720. 
(32) Honda, K.; Morita, M.; Otsuka, H.; Takahara, A. Molecular Aggregation Structure and Surface Properties of Poly(Fluoroalkyl Acrylate) Thin Films. Macromolecules 2005, 38, 5699-5705.

(33) Honda, K.; Morita, M.; Sakata, O.; Sasaki, S.; Takahara, A. Effect of Surface Molecular Aggregation State and Surface Molecular Motion on Wetting Behavior of Water on Poly(Fluoroalkyl Methacrylate) Thin Films. Macromolecules 2009, 43, 454-460.

(34) Stachewicz, U.; Peker, I.; Tu, W.; Barber, A. H. Stress Delocalization in Crack Tolerant Electrospun Nanofiber Networks. ACS Appl. Mater. Interfaces 2011, 3, 1991-1996.

(35) Hang, F.; Lu, D.; Bailey, R. J.; Jimenez-Palomar, I.; Stachewicz, U.; Cortes-Ballesteros, B.; Davies, M.; Zech, M.; Boedefeld, C.; Barber, A. H. In Situ Tensile Testing of Nanofibers by Combining Atomic Force Microscopy and Scanning Electron Microscopy. Nanotechnology 2011, 22, 365708-8.

(36) Stachewicz, U.; Hang, F.; Barber, A. H. Adhesion Anisotropy between Contacting Electrospun Fibers. Langmuir 2014, 30, 6819-25.

(37) Brewer, S. A.; Willis, C. R. Structure and Oil Repellency: Textiles with Liquid Repellency to Hexane. Appl. Surf. Sci. 2008, 254, 6450-6454.

(38) Grill, A. Cold Plasma in Materials Fabrication: From Fundamentals to Applications; IEEE Press: New York, 1994.

(39) Yazdanpanah, M. M.; Hosseini, M.; Pabba, S.; Berry, S. M.; Dobrokhotov, V. V.; Safir, A.; Keynton, R. S.; Cohn, R. W. Micro-Wilhelmy and Related Liquid Property Measurements Using Constant-Diameter Nanoneedle-Tipped Atomic Force Microscope Probes. Langmuir 2008, 24, 13753-13764.

(40) Sader, J. E.; Chon, J. W. M.; Mulvaney, P. Calibration of Rectangular Atomic Force Microscope Cantilevers. Rev. Sci. Instrum. 1999, 70, 3967-3969.

(41) Bushby, A. J.; P'ng, K. M. Y.; Young, R. D.; Pinali, C.; Knupp, C.; Quantock, A. J. Imaging Three-Dimensional Tissue Architectures by Focused Ion Beam Scanning Electron Microscopy. Nat. Protoc. 2011, 6, 845-858.

(42) Bailey, R. J.; Geurts, R.; Stokes, D. J.; de Jong, F.; Barber, A. H. Evaluating Focused Ion Beam Induced Damage in Soft Materials. Micron 2013, 50, 51-56. 
Figures (displays)

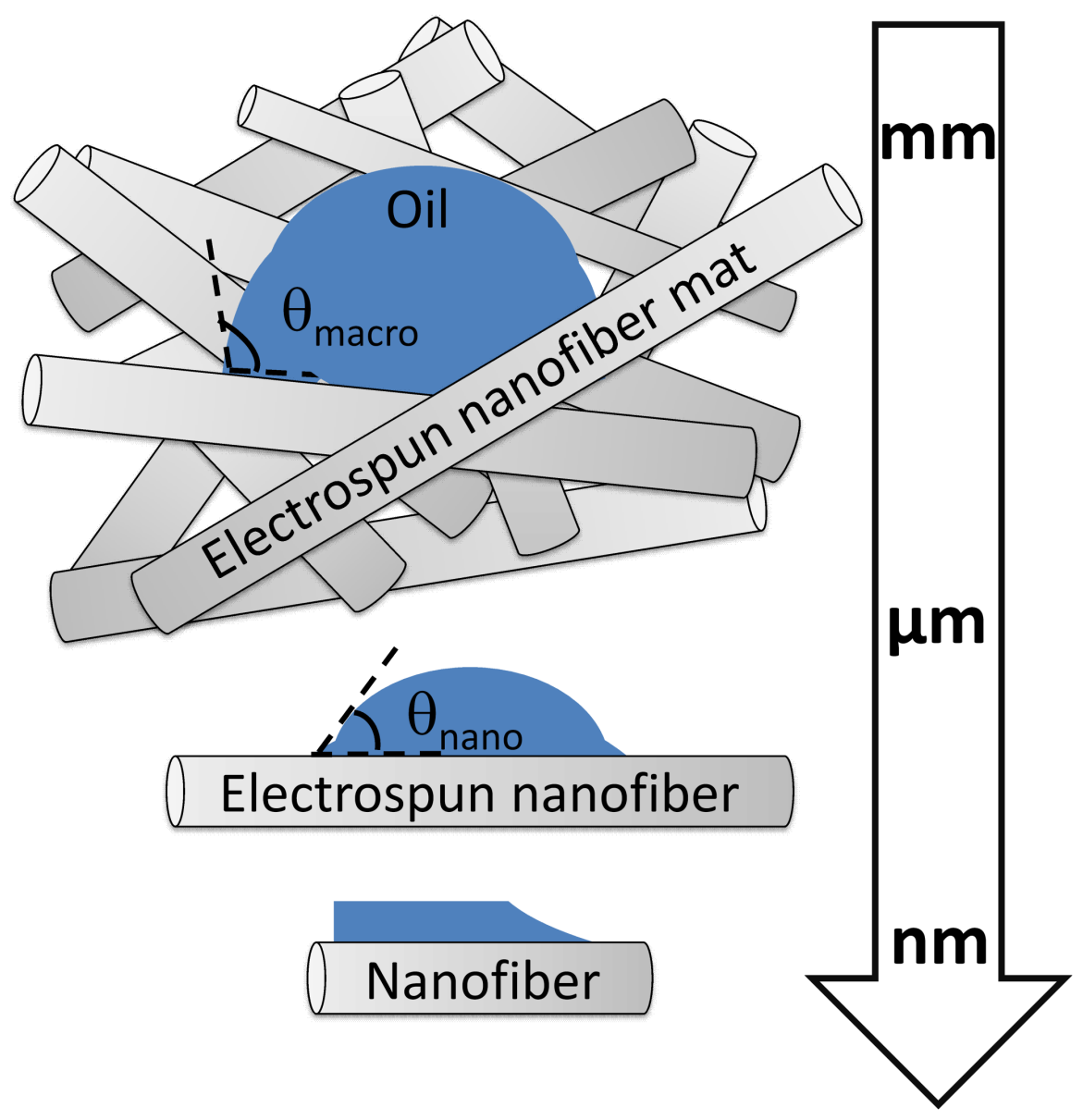

Figure 1. Schematic of wetting hierarchy in electrospun nanofiber networks. 

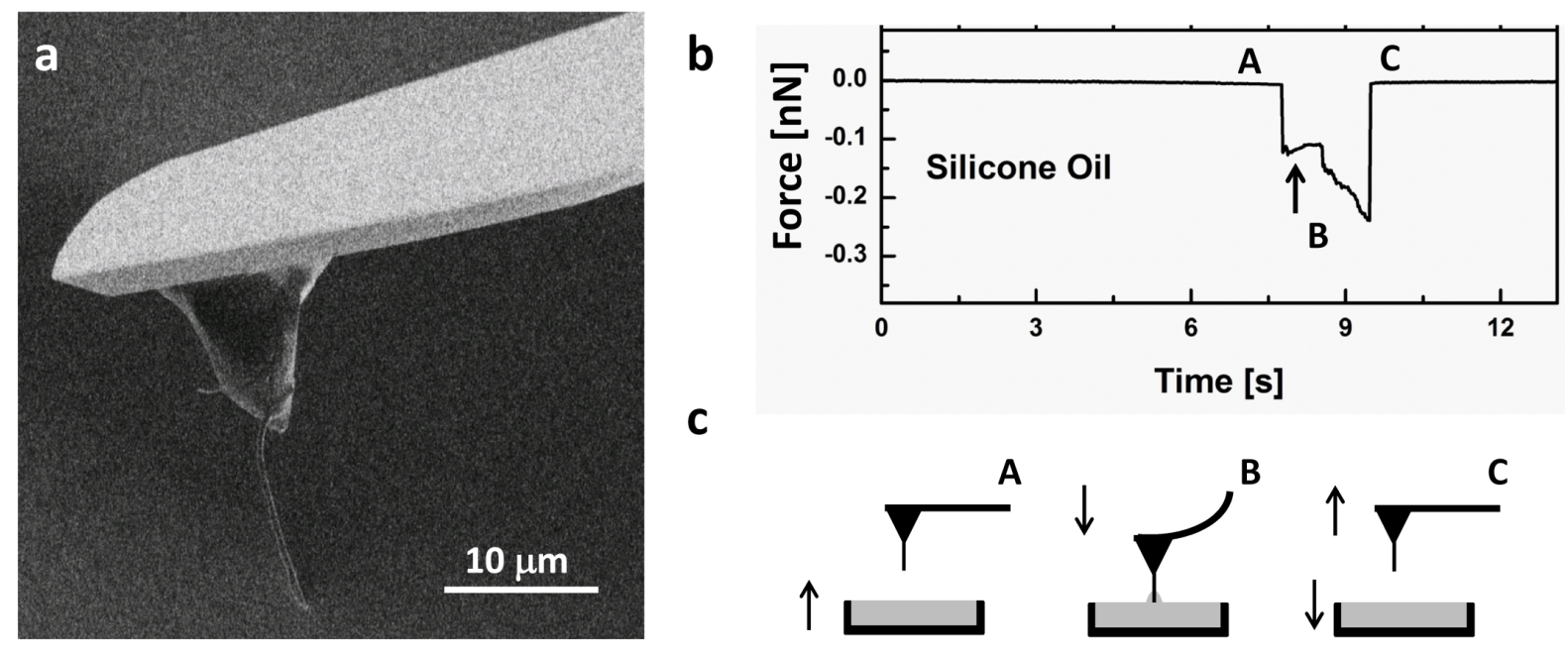

C

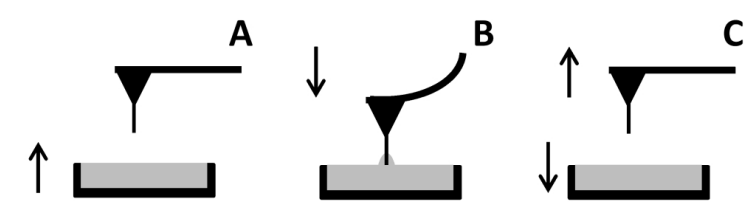

Figure 2. a) SEM image of an isolated PA6 nanofiber, with average diameter of $480 \mathrm{~nm}$, attached to the AFM tip after sectioning with FIB. b) Plot of the AFM cantilever deflection (DFL), converted to force, during time progression of the wetting experiment. AFM cantilever deflection is constant during the initial part of the experiment as the end of the electrospun nanofiber is above the liquid surface (A). An abrupt drop in the AFM cantilever deflection occurs when the nanofiber contacts the liquid surface during wetting of the nanofiber (B). Once the nanofiber is remove from the liquid, the cantilever deflection corresponding to applied force returns to the constant position again (C). Please note the magnitude of the wetting forces acting on the electrospun nanofiber is a function of the magnitude of the AFM cantilever deflection. c) Schematic of the wetting steps and force acting on the AFM cantilever $(A, B, C)$ with attached nanofiber. 

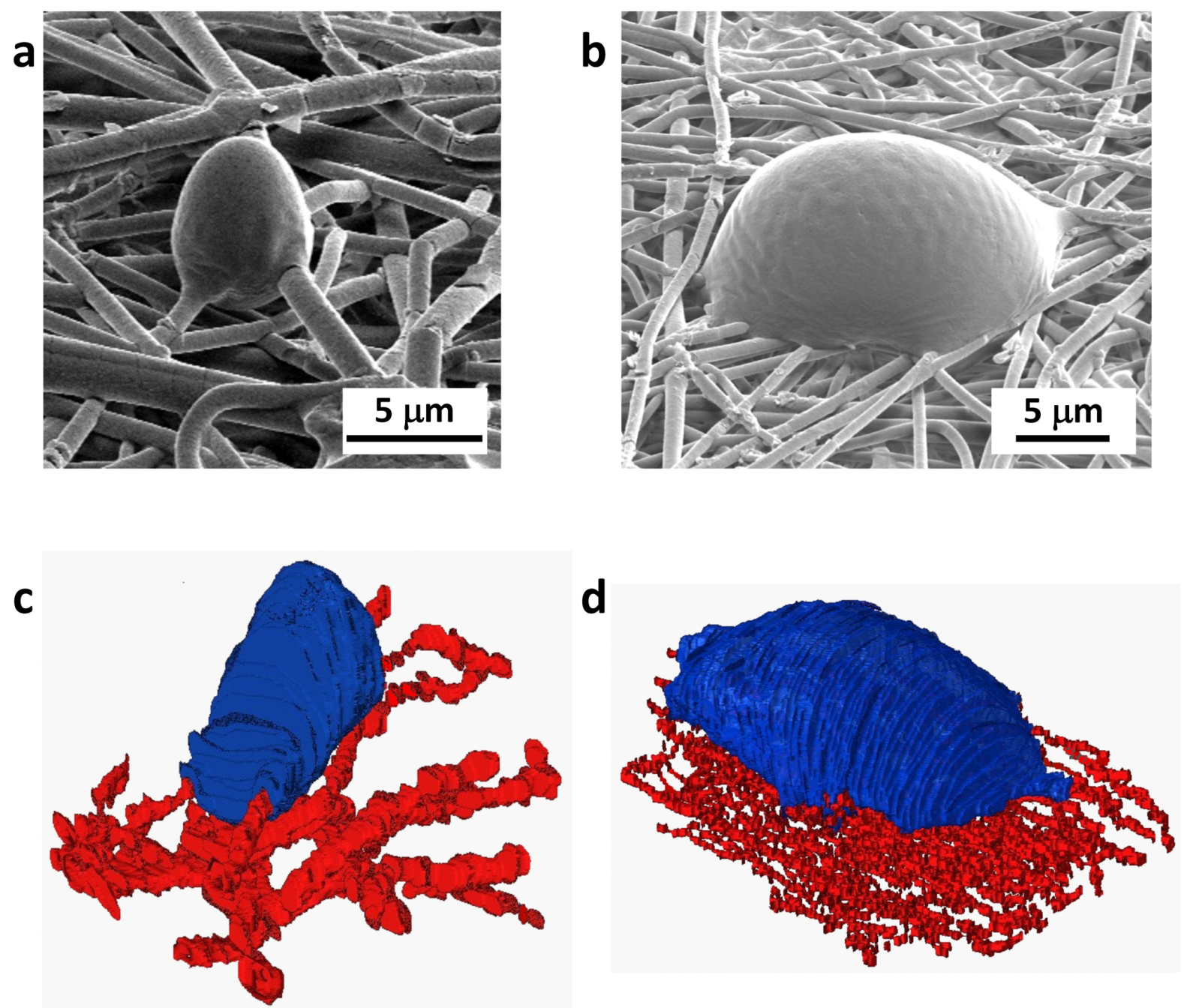

Figure 3. 2D cryo-SEM micrographs in a) and b) showing individual silicone oil droplets before slice and view imaging. 3D reconstructions of the individual droplets in c) and d) showing silicone oil droplets (in blue) on a plasma treated electrospun nanofiber array (shown in red). 
a

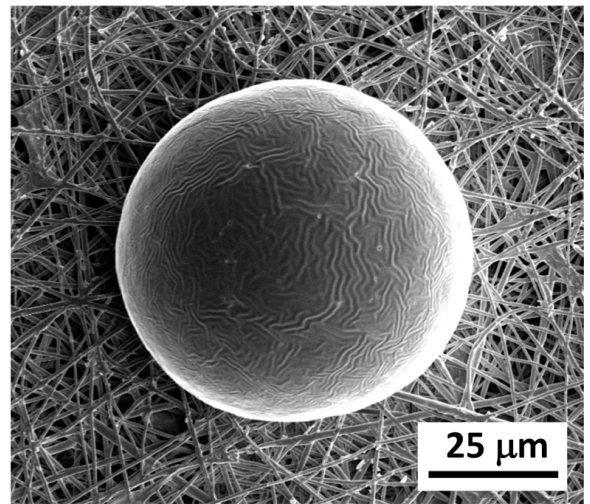

b
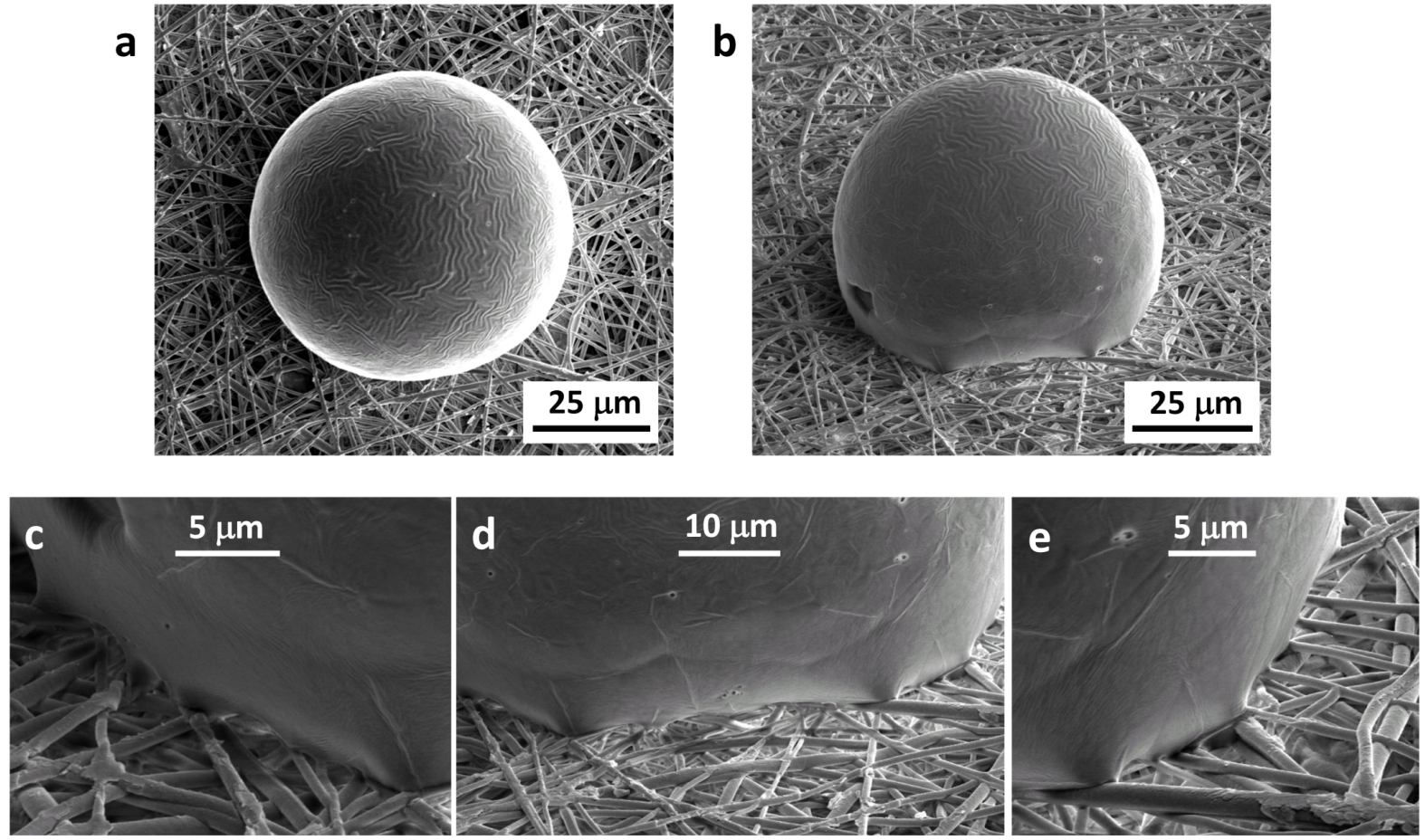

Figure 4. 2D cryo-SEM micrographs of sprayed silicone oil on plasma treated electrospun nylon 6 nanofibers in a) and b). Higher magnification cryo-SEM imaging in c), d) and e) indicates the contact line between silicone oil and nanofibers 
a

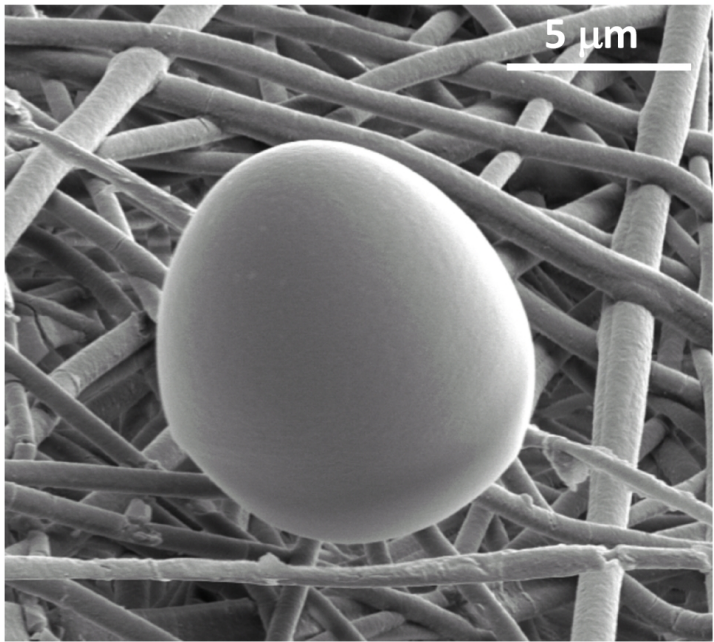

C

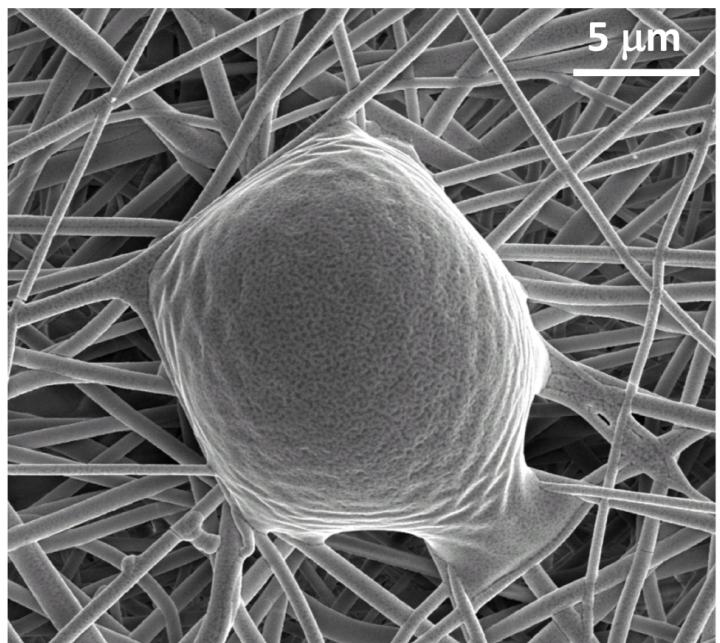

b

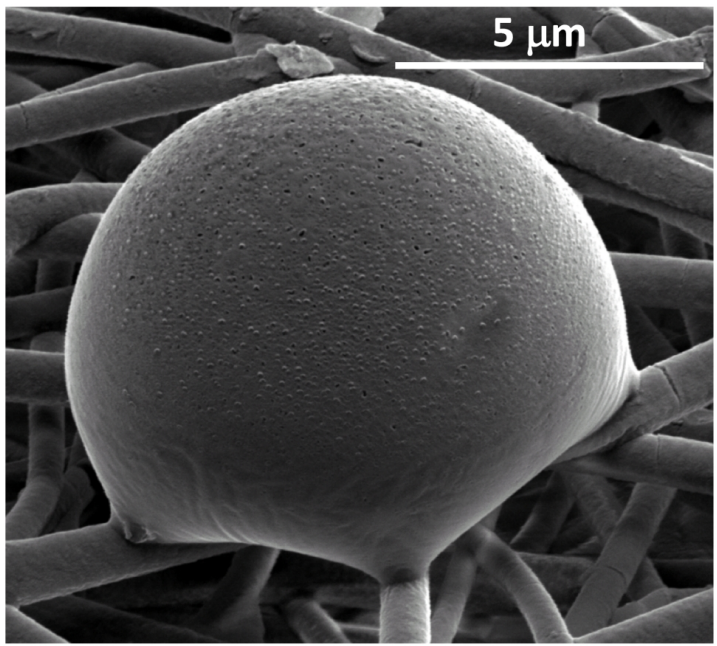

d

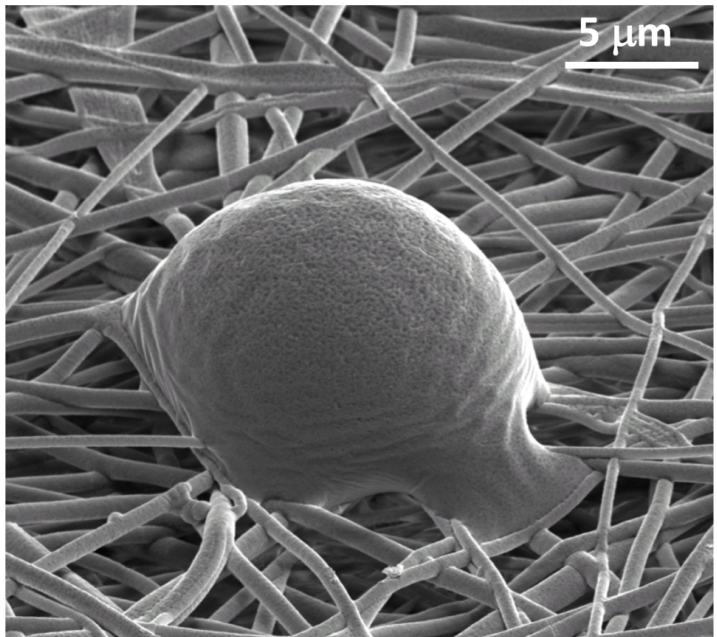

Figure 5. Scanning electron micrographs of silicone oil droplets contacting nanofibers network of plasma treated electrospun fibers. a) side view of a non-wetting droplet suspended on nanofibers, b) side view of an oil droplet with wetting contact points on individual nanofibers with $\mathbf{c}$ ) top view and d) side view of a further oil droplet wetting nanofibers. 


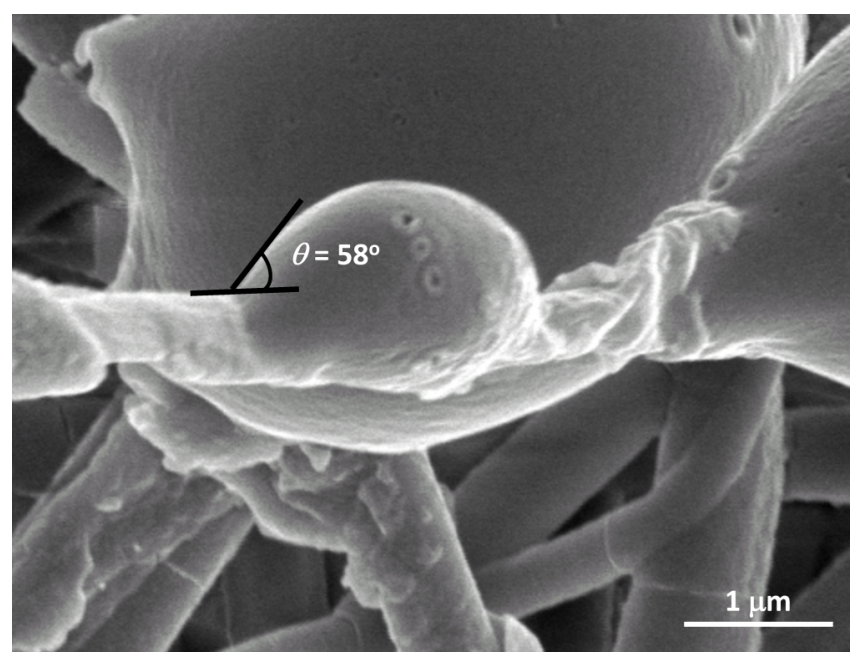

Figure 6. Scanning electron micrograph of a relatively small silicone oil droplet contacting an individual plasma treated electrospun nanofiber. Contact angles indicate partial wetting that was consistent with Wilhelmy balance measurements.

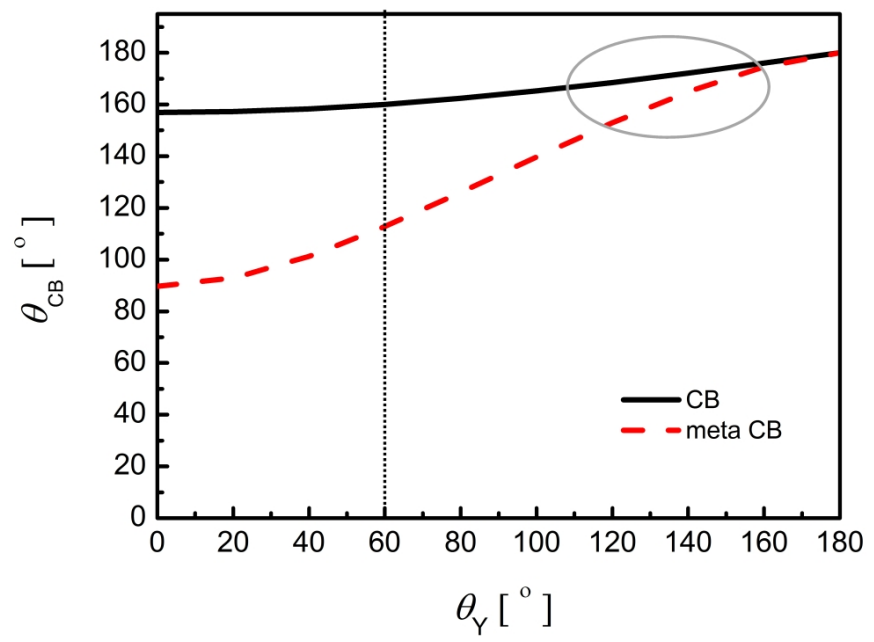

Figure 7. Plot of a liquid contact angle on a porous surface, described by the liquid fraction in contact with air $f_{2}$, equal to 0.96 (or fraction of nanofibers $f_{2}=0.04$ ) and $\theta_{Y}=\theta_{\text {nano }}=60^{\circ}$ for silicone oil on nylon 6 nanofibers, indicated with a dotted line. The superoleophobic region where $\theta_{C B} \geq 150^{\circ}$ is circled at the top right corner of the plot. 
Table 1. Wetting data for the contact between plasma coated electrospun PA6 nanofibers and silicone oil, with the contact angle $\boldsymbol{\theta}_{\text {nano }}$ calculated from Equation 2.

\begin{tabular}{lccc}
\hline Probe Liquid & Wetting Force $[\mathrm{nN}]$ & $\operatorname{Cos} \boldsymbol{\theta}_{\text {nano }}$ & $\boldsymbol{\theta}_{\text {nano }}\left[^{\circ}\right]$ \\
\hline Silicone Oil & 12.03 & 0.53 & 58.24 \\
& 11.54 & 0.51 & 59.65 \\
11.61 & 0.51 & 59.43 \\
11.02 & 0.48 & 61.15 \\
& 11.47 & 0.50 & 59.84 \\
\hline & & Average $\boldsymbol{\theta}_{\text {nano }}\left[^{\circ}\right]$ & $\mathbf{5 9 . 6 6}$ \\
\hline & & Standard Deviation & $\mathbf{2 . 0 8}$ \\
\hline
\end{tabular}

